\title{
Bursting and Synchronization of Coupled Neurons under Electromagnetic Radiation
}

\author{
Xiaoyu Hu $\mathbb{D}^{1}$ and Chongxin Liu ${ }^{2}$ \\ ${ }^{1}$ School of Electronics and Information, Northwestern Polytechnical University, Xian 710072, China \\ ${ }^{2}$ School of Electrical Engineering, Xian Jiaotong University, Xian 710049, China \\ Correspondence should be addressed to Xiaoyu Hu; huxiaoyucool@163.com
}

Received 12 May 2019; Revised 11 July 2019; Accepted 6 August 2019; Published 4 December 2019

Guest Editor: Lazaros Moysis

Copyright (C) 2019 Xiaoyu Hu and Chongxin Liu. This is an open access article distributed under the Creative Commons Attribution License, which permits unrestricted use, distribution, and reproduction in any medium, provided the original work is properly cited.

\begin{abstract}
Bursting is an important firing activity of neurons, which is caused by a slow process that modulates fast spiking activity. Based on the original second-order Morris-Lecar neuron model, an improved third-order Morris-Lecar neuron model can produce bursting activity is proposed, in which the effect of electromagnetic radiation is considered as a slow process and the original equation of Morris-Lecar neuron model as a fast process. Extensive numerical simulation results show that the improved neuron model can produce different types of bursting, and bursting activity shows a deep dependence on system parameters and electromagnetic radiation parameters. In addition, synchronization transitions of identical as well as no-identical coupled third-order MorrisLecar neurons are studied, the results show that identical coupled neurons experience a complex synchronization process and reach complete synchronization finally with the increase of coupling intensity. For no-identical coupled neurons, only anti-phase synchronization and in-phase synchronization can be reached. The studies of bursting activity of single neuron and synchronization transition of coupled neurons have important guiding significance for further understanding the information processing of neurons and collective behaviors in neuronal network under electromagnetic radiation environment.
\end{abstract}

\section{Introduction}

The biological or human neural system is usually composed of millions of neurons, which can generate, transmit, receive, and process information by firing various types of electrical activities. Since the pioneering work of Hodgkin and Huxley [1], many models have been proposed for modelling and simulating the electrical activities of a neuron [2-9]. For a neuron, spiking and bursting are two major categories, and they may be periodic motion or chaotic motion. Indeed, bursting is considered as neuron activity alternates between a quiescent state and repetitive spiking, and it is a dynamical consequence of fast/slow dynamics. Bursting is an important firing pattern, and it has been confirmed that neurons in different regions of brain produce bursting activities [10].

Neurons are sensitivity to many external factors, and electrical activities of a neuron and collective behaviours in neuronal network will be changed under certain conditions. Time delay [11-17], noise [18, 19], and network topology [20-23] are common factors being considered to investigate firing behaviours of neuron and collective behaviours in neuronal network. It is worth noting that electromagnetic radiation is another one cannot be ignored. With the development of modern industry, wide utilizations of electric equipment make neural system are exposed to an environment full of electromagnetic radiation, which has a great influence on the dynamics of a single neuron and network of neurons. In Ref. [24], Wang et al. suggested that the strong external electromagnetic field facilitates the neuron firing action potentials and enhances the mean firing rate of the network, but disrupts the synchronicity of the activities of the neural network. In Ref. [25], Li et al. developed a mathematical model to describe the effect of electromagnetic radiation, the results show that electrical activities of a single neuron can be suppressed by electromagnetic radiation, and spatiotemporal patterns in neuronal network are also suppressed from the stable propagating wave state to a homogeneous resting state. Rebertson et al. [26] argued that low-frequency pulsed electromagnetic field exposure can alter neuroprocessing in humans. In Ref. [27], a small Hopfield neural network with the electromagnetic radiation 


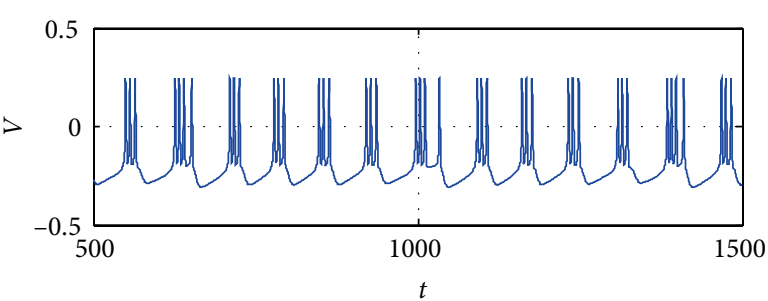

(a)

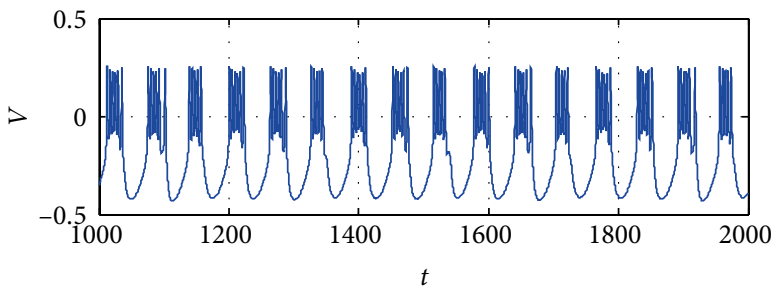

(c)

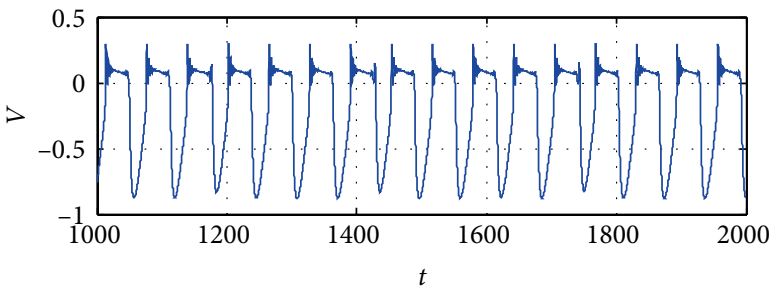

(e)

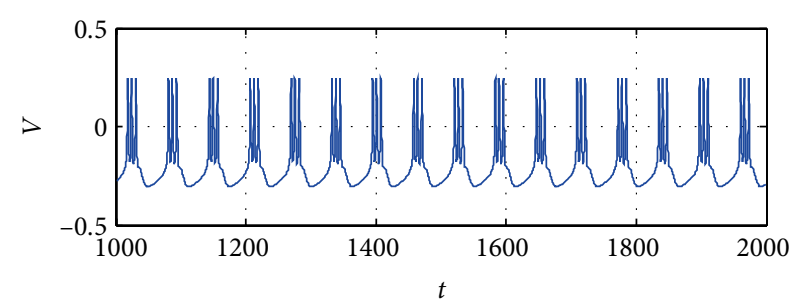

(b)

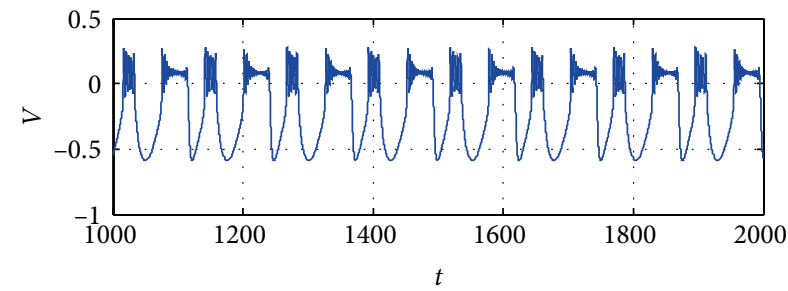

(d)

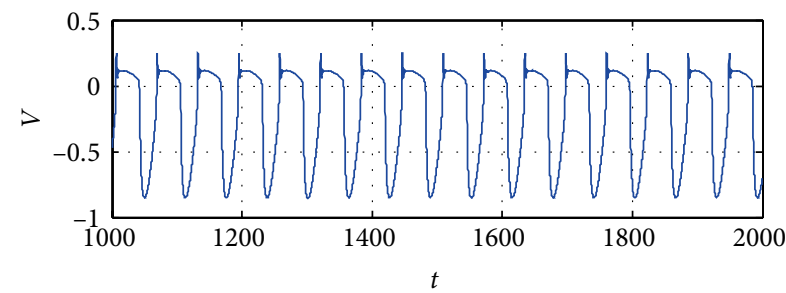

(f)

FIGURE 1: Sampled time series for membrane potential when the intensity of external forcing currents is chosen as different values with fixed angular frequency $\omega=0.1$. (a) $I=0.001$, (b) $I=0.005$, (c) $I=0.05$, (d) $I=0.1$, (e) $I=0.2$, and (f) $I=0.5$. The effect of electromagnetic radiation is considered by setting $k_{1}=0.2, k_{2}=0.15$, and $k_{3}=0.15$.

being considered is constructed, in which the previous steady neural network can present abundant chaotic dynamics, and hidden attractors can be observed.

Memory is a natural characteristic of neuron, and it has been considered in studies from neuron models to collective behaviours in neuronal network recently. For example, references [28-30] have proved that the ionic channels of neuron models, e.g., Hodgkin-Huxley and Morris-Lecar neuron model, have memory effect and they can be substituted by first-order or second-order memristors. Moreover, memristive relation is also used to stress the memory effect in some memristor-based neural network [31-33]. Indeed, memristor is an effective element to characteristic the memory effect in neuron and network of neurons. From this point of view, the effect of electromagnetic radiation on neuron can be considered as a variation of magnetic flux, and the flux-controlled memristor is available to represent the memory effect of magnetic flux. As a result, Ma et al. [34-36] proposed several models to describe the effect of electromagnetic radiation on the electrical activities of neuron by using magnetic flux, in which a memristor-like feedback is employed to realize coupling between magnetic flux and membrane potential. It is found that multiple modes of spiking activities can be observed. Moreover, synchronization, noise effect, and spatiotemporal dynamics in neuron and neural networks under electromagnetic radiation were also investigated [37-41]. The effect of electromagnetic radiation can be described by time-varying magnetic flux, the coupling of electromagnetic field between neurons can be described by exchange of magnetic flux as well, which results in another effective way for coupling between neurons, i.e., field coupling. In Refs. [42-44], field coupling rather than synaptic coupling is considered as a coupling mode between neurons and neural networks, it is found that multiple modes of synchronization can be observed from coupled neurons or neural networks.

In this paper, we propose an improved Morris-Lecar neuron model with electromagnetic radiation being considered, in which the fluctuation of electromagnetic radiation is described by using magnetic flux and considered as a slow subsystem. Unlike previous models, multiple modes of bursting activities are observed. Furthermore, synchronization transitions in coupled identical bursting neurons as well as no-identical bursting neurons are studied. The organization of this paper is as follows. In Section 2, the model setting and description is introduced. In Section 3, numerical results are discussed and analysed carefully. Section 4 summarizes and concludes this paper.

\section{Model Setting and Description}

Bursting activities are results of fast/slow dynamics, and they cannot be observed in original two-dimensional Morris-Lecar neuron model under constant external forcing current. Researchers [5, 45-47] have explored several improved Morris-Lecar neuron model, in which external forcing current 


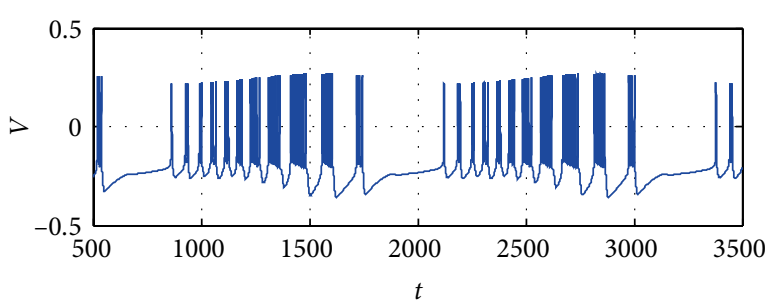

(a)

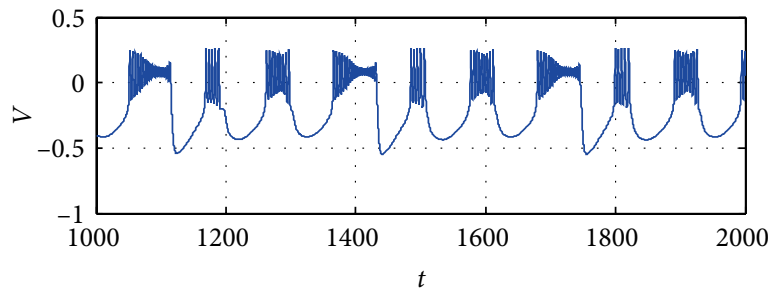

(c)

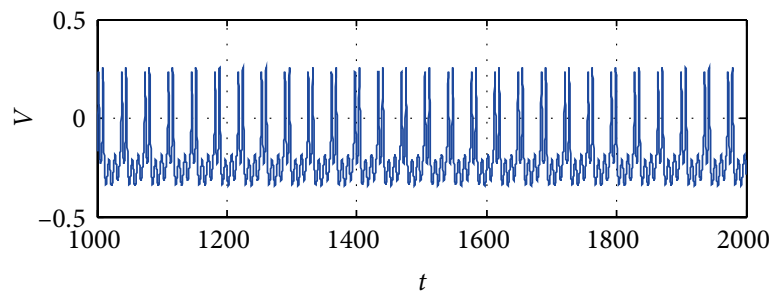

(e)

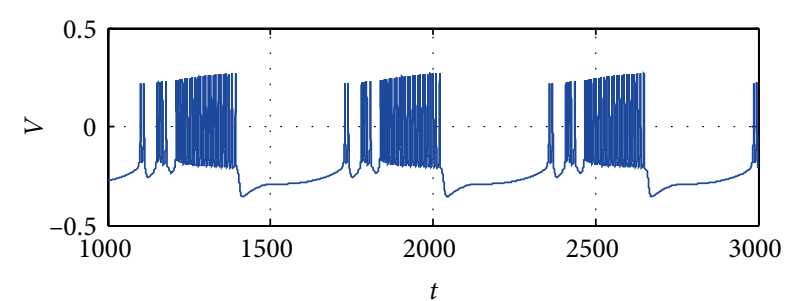

(b)

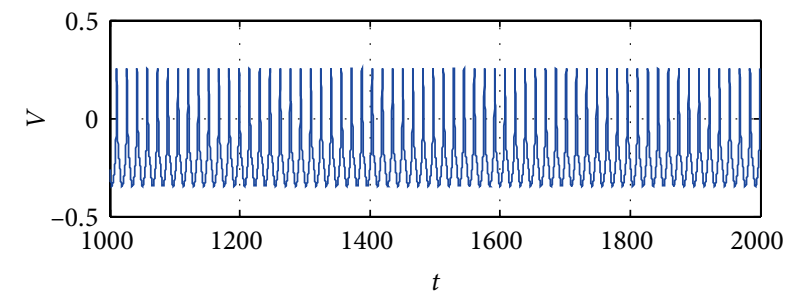

(d)

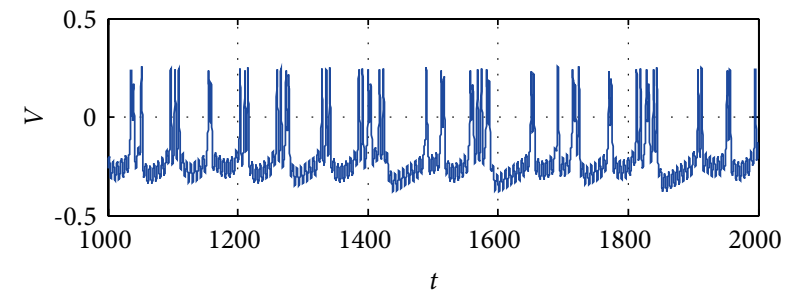

(f)

FIGURE 2: Sampled time series for membrane potential when the angular frequency of external forcing currents is chosen as different values with fixed intensity $I=0.05$. (a) $\omega=0.005$, (b) $\omega=0.01$, (c) $\omega=0.06$, (d) $\omega=0.4$, (e) $\omega=0.7$, and (f) $\omega=1.0$. The effect of electromagnetic radiation is considered by setting $k_{1}=0.2, k_{2}=0.15$, and $k_{3}=0.15$.

is considered as a varied state variable with a very slow rate, and several types of bursting are obtained. Inspired by Refs. $[35,48-50]$, electromagnetic radiation is considered when improved Morris-Lecar neuron model is constructed, in which the effect of electromagnetic radiation is regarded as a slow subsystem and two-dimensional Morris-Lecar neuron model as a fast subsystem. As a result, the improved Morris-Lecar neuron model is described as follows.

$$
\begin{aligned}
\dot{V}= & -g_{C a} M_{\infty}(V)\left(V-V_{C a}\right)-g_{K} W\left(V-V_{K}\right) \\
& -g_{l}\left(V-V_{l}\right)-I_{s t}+k_{1}\left(\alpha+\beta \varphi^{2}\right) V, \\
\dot{W}= & \lambda(V)\left(W_{\infty}(V)-W\right), \\
\dot{\varphi}= & k_{2} V+k_{3} \varphi_{\text {ext }},
\end{aligned}
$$

where

$$
\begin{aligned}
M_{\infty}(V) & =\frac{1}{2}\left(1+\tanh \frac{V-V_{1}}{V_{2}}\right), \\
W_{\infty}(V) & =\frac{1}{2}\left(1+\tanh \frac{V-V_{3}}{V_{4}}\right), \\
\lambda(V) & =\frac{1}{3} \cosh \left(\frac{V-V_{3}}{2 V_{4}}\right) .
\end{aligned}
$$

In this model, $V$ represents membrane potential, $W$ represents recovery variable, $\varphi$ is magnetic flux which is a slower variable than $V$ and $W . V_{C a}, V_{K}$, and $V_{l}$ are steady state potentials for calcium, potassium, and leak ion channels, respectively. $M_{\infty}(V)$ and $W_{\infty}(V)$ define the stable values of opening probability for calcium and potassium, where $V_{1}, V_{2}, V_{3}$, and $V_{4}$ are

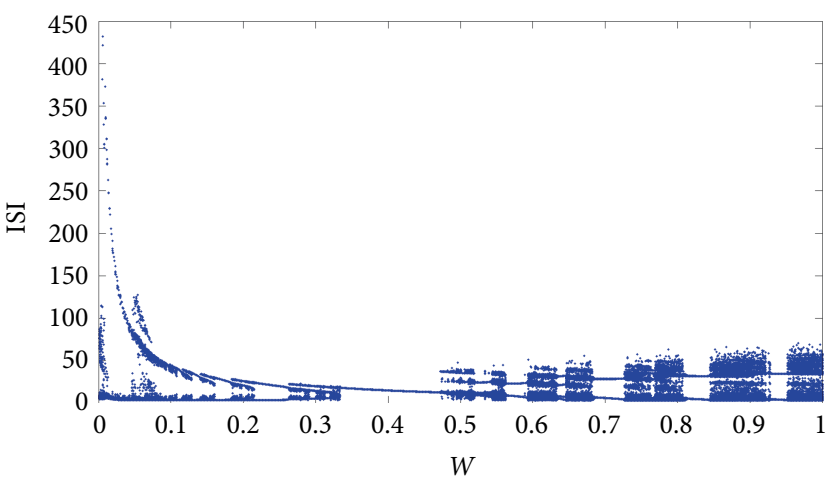

FIGURE 3: Bifurcation diagram of ISI by setting angular frequency $\omega$ as the bifurcation parameter with $I=0.05$. The effect of electromagnetic radiation is considered by setting $k_{1}=0.2, k_{2}=0.15$, and $k_{3}=0.15$.

parameters of steady states. $I_{s t}$ is external forcing current and $I_{s t}=I \sin (\omega t)$. The term $k_{1}\left(\alpha+\beta \varphi^{2}\right) V$ defines the feedback current on membrane potential when magnet flux is changed in media, and $k_{1}$ is the feedback gain. $k_{2}$ is a factor which describes the contribution of varied magnetic flux on the formation of membrane potential. $k_{3} \varphi_{\text {ext }}$ is considered as leakage magnet flux, and $\varphi_{\text {ext }}$ is chosen as a constant value 0.2 . The rest system parameter are selected as $g_{C a}=1.2, g_{K}=2, g_{l}=0.5$, $V_{C a}=1, V_{l}=-0.5, V_{K}=-0.7, V_{1}=-0.01, V_{2}=0.15, V_{3}=0.1$, $V_{4}=0.05, \alpha=0.1$, and $\beta=-0.09$. 


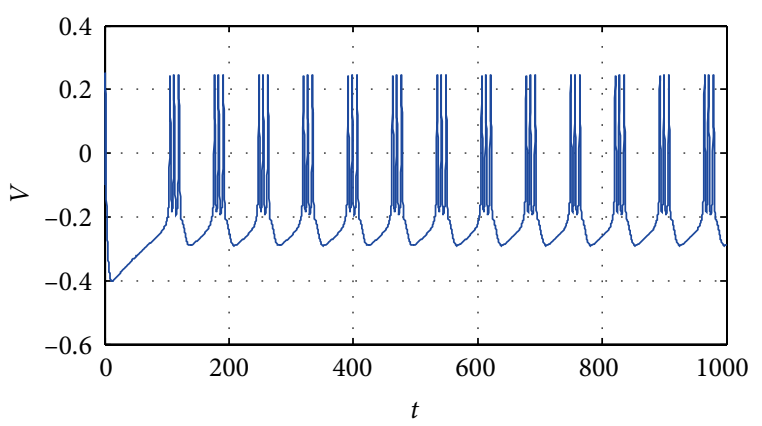

(a)

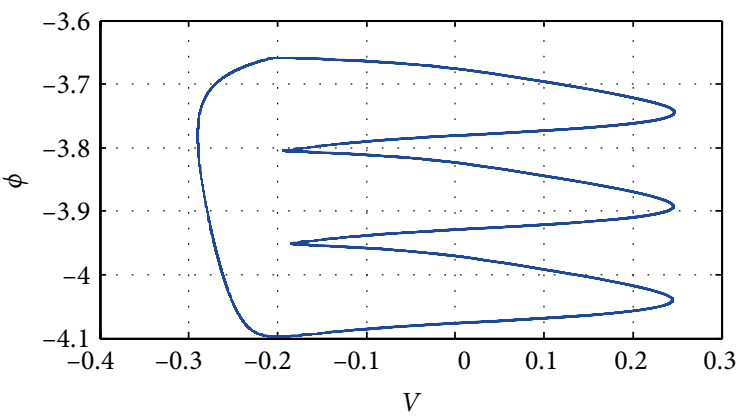

(c)

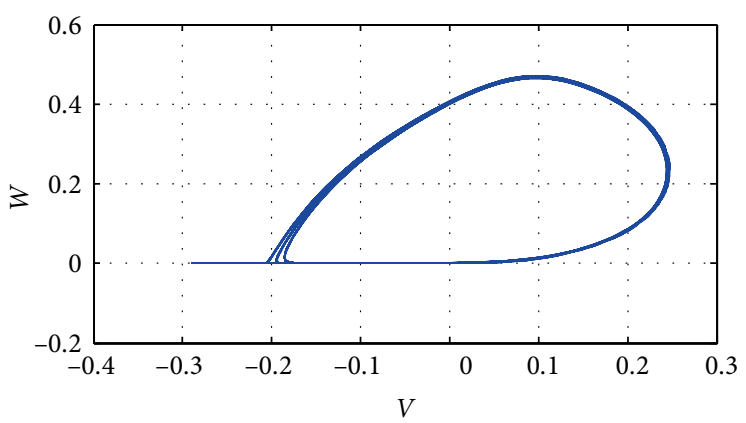

(b)

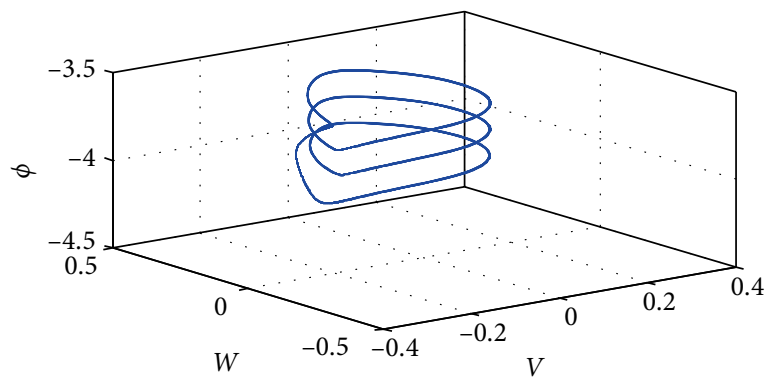

(d)

FIGURE 4: Sampled time series for membrane potential and phase diagrams when the system parameters are selected as $I=0, k_{1}=0.2$, $k_{2}=0.15$, and $k_{3}=0.15$.

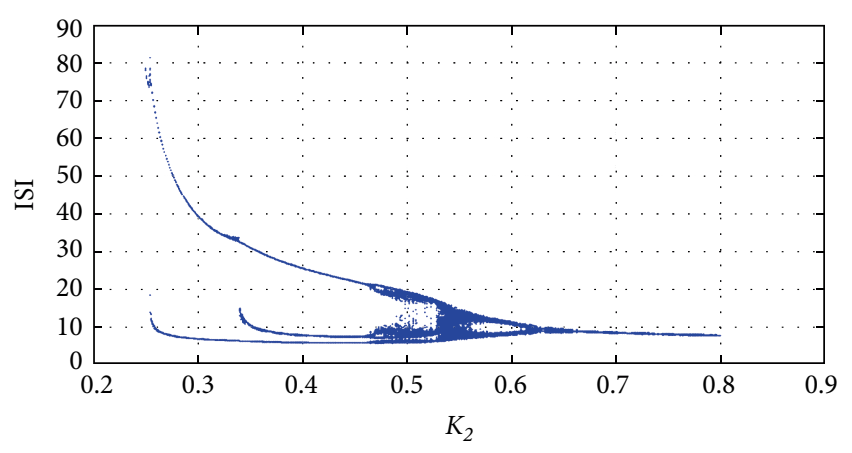

FIGURE 5: Bifurcation diagram of ISI by setting $k_{2}$ as the bifurcation parameter with $I=0, k_{1}=0.2$, and $k_{3}=0.3$.

\section{Numerical Results and Discussion}

3.1 Bursting in Improved ML Neuron Model under Electromagnetic Radiation. In this section, fourth order Runge-Kutta algorithm is used with time step $h=0.01$. The initial values are set as $\left(V_{0}, W_{0}, \varphi_{0}\right)=(-0.1,0,-2.5)$. At first, sampled time series for membrane potential are detected with different intensity of external forcing current, and system parameters and angular frequency are selected as $k_{1}=0.2$, $k_{2}=0.15, k_{3}=0.15$, and $\omega=0.1$. The results are shown in Figure 1.

It is found that even a very small intensity of external forcing current can trigger bursting activities of neuron, and they can be either chaotic or periodic, as show in Figures 1(a)-1(c). Particularly, the model can present two types of burst alternately with appropriate intensity of external forcing current, as shown in Figure 1(d). With the increase of external current intensity, the emerging type of bursting is stay behind. Furthermore, sampled time series for membrane potential are calculated with different angular frequency of external forcing currents being used, and the results are shown in Figure 2.

In numerical simulations, the intensity of external forcing current is fixed at $I=0.05$, and the effect of electromagnetic radiation is considered by setting $k_{1}=0.2, k_{2}=0.15$, and $k_{3}=0.15$. When small angular frequency is used, the model presents a kind of cluster bursting, i.e., each cluster consist of several bursts which have different number of spikes, as shown in Figures 2(a) and 2(b). In Figure 2(c) it is worth noting that three types of burst emerge alternately when the angular frequency is selected as $\omega=0.06$. Moreover, spiking, periodic bursting, and chaotic bursting can be also observed when angular frequency is chosen appropriately, as shown in Figures 2(d)-2(f), respectively. To better understand the effect of angular frequency on electrical activities of neurons, bifurcation analysis is carried out by calculating the interspike interval (ISI), and the results are shown in Figure 3. It is found that the neuron can present bursting behaviours in a large parameter region, and chaotic bursting, and periodic bursting emerge alternately with the increase of angular frequency.

In order to discern the effect of electromagnetic radiation, external forcing current is set as $I=0$, and sampled time series for membrane potential and phase diagrams when the system parameters are selected $k_{1}=0.2, k_{2}=0.15$, and $k_{3}=0.15$ are shown in Figure 4. In Figure 4, period-3 bursting is obtained, and it belongs to a type of "Flod/Homoclinic bursting" according to the classification methods in Ref. [45]. To better understand the effect of system parameters on firing activities of 


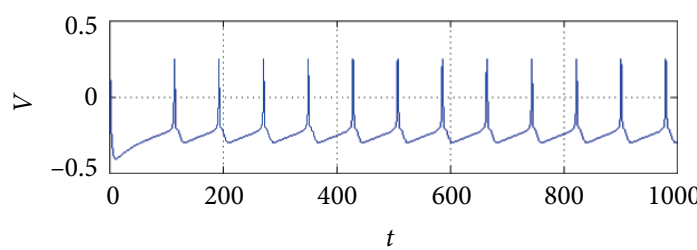

(a)

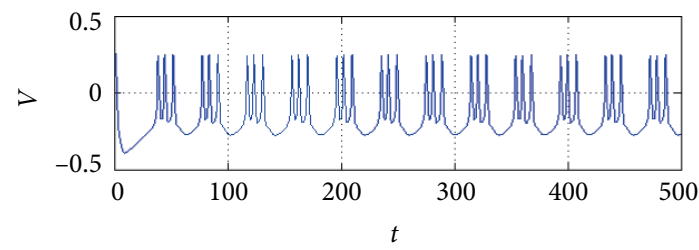

(c)

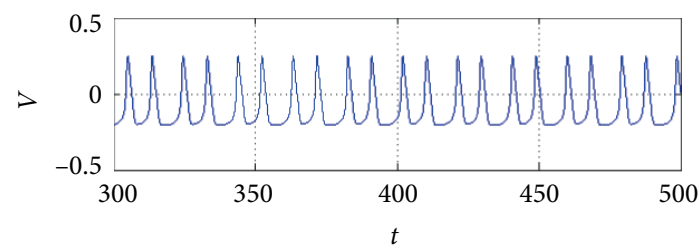

(e)

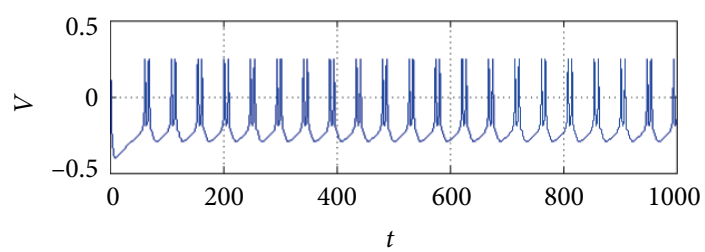

(b)

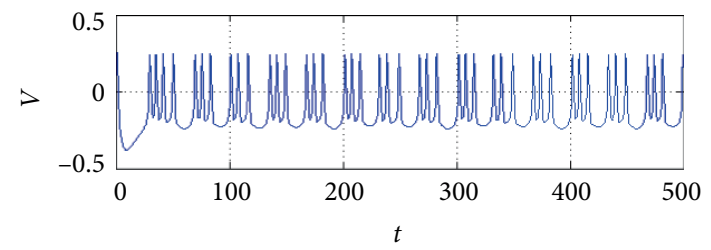

(d)

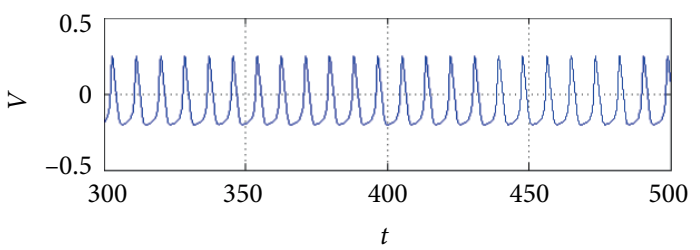

(f)

Figure 6: Sampled time series for membrane potential when $k_{2}$ are chosen as different values with $I=0, k_{1}=0.2$, and $k_{3}=0.3$. (a) Spiking with $k_{2}=0.25$, (b) period-2 bursting with $k_{2}=0.3$, (c) period-3 bursting with $k_{2}=0.4$, (d) chaotic bursting with $k_{2}=0.5$, (e) period-2 bursting with $k_{2}=0.6$, and (f) spiking with $k_{2}=0.7$.

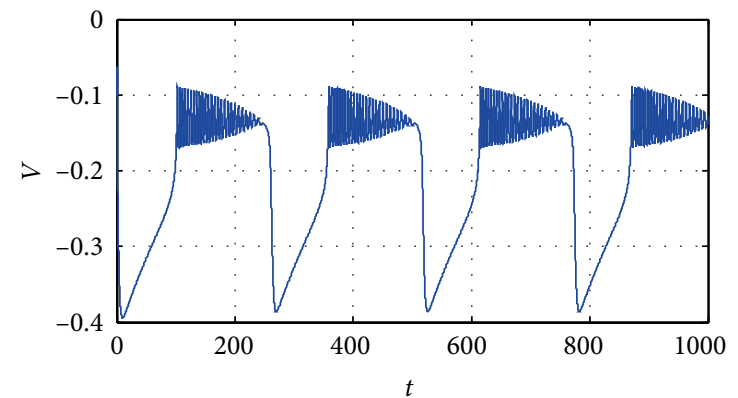

(a)

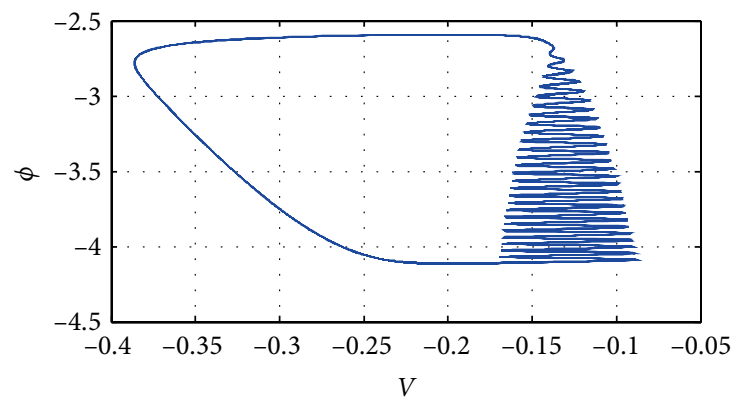

(c)

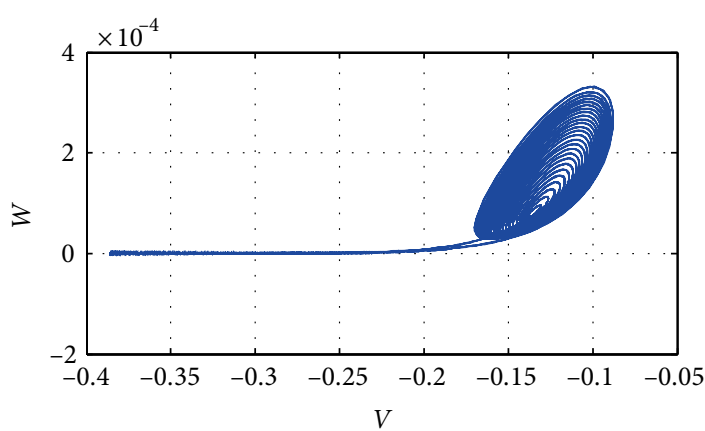

(b)

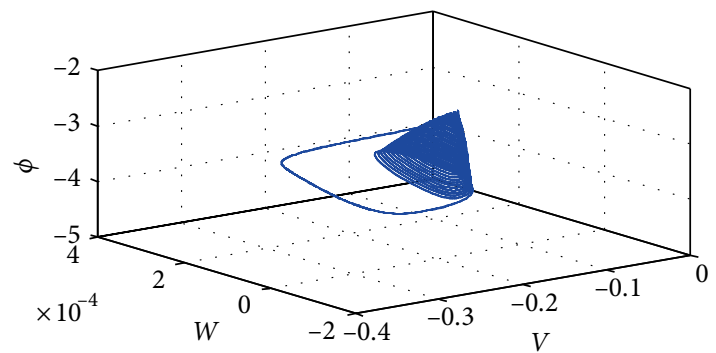

(d)

FIGURE 7: Sampled time series for membrane potential and phase diagrams when the system parameters are selected as $I=0, k_{1}=0.2$, $k_{2}=0.15, k_{3}=0.15$, and $V_{K}=-300 \mathrm{mV}$.

neuron, $k_{2}$ is selected as bifurcation parameter and bifurcation diagram is calculated, and the results are shown in Figure 5.

In Figure 5, it is found that each burst can have more spikes when $k_{2}$ is less than 0.46 , and increased $k_{2}$ is accompanied by a chaotic bursting activity. When parameter $k_{2}$ exceeds 0.63 , only spiking activities can be observed. Particularly, the change of spike number in each burst with the increase of $k_{2}$ experiences a parameter region that chaotic bursting emerges. 


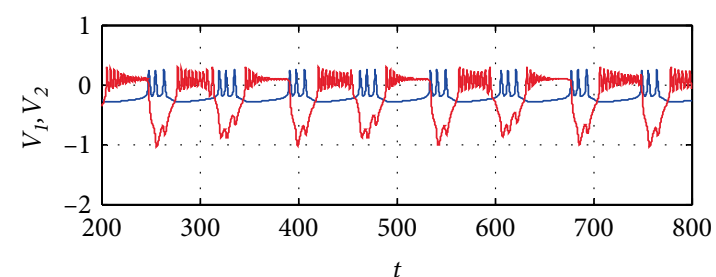

(a)

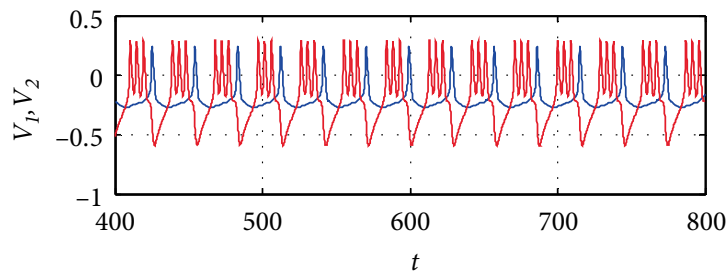

(c)

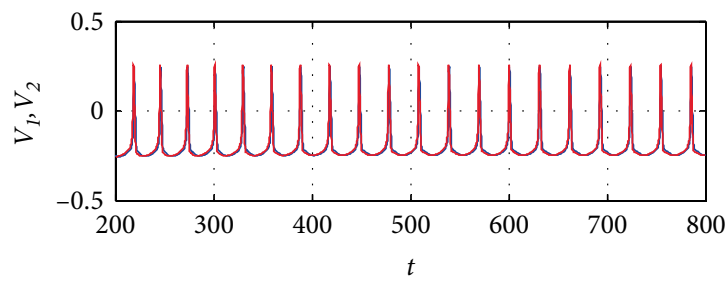

(e)

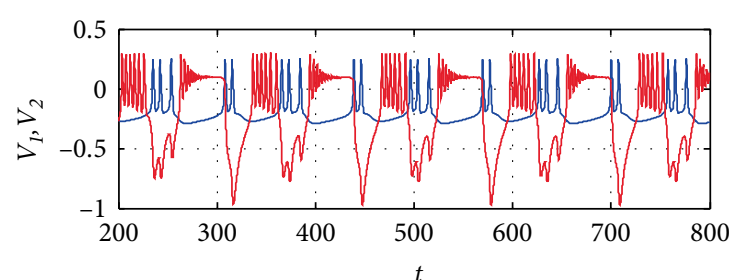

(b)

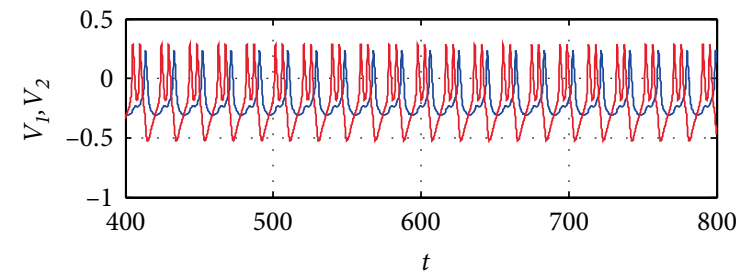

(d)

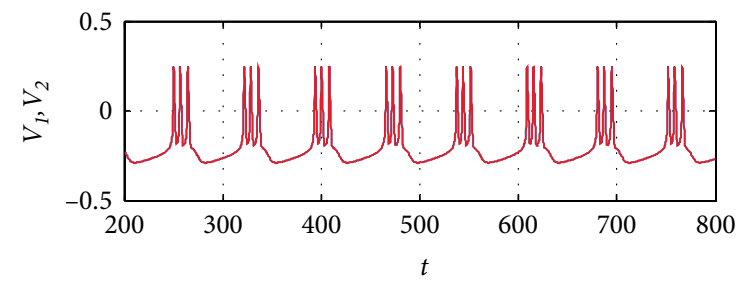

(f)

FIGURE 8: Synchronization transition of two identical coupled neurons with different coupling intensity values. (a) $g_{s}=0.001$, (b) $g_{s}=0.004$, (c) $g_{s}=0.025$, (d) $g_{s}=0.09$, (e) $g_{s}=0.31$, and (f) $g_{s}=1.0$. Initial conditions are selected as $(-0.1,0,-2.5)$ and $(-0.2,0,-2.5)$, respectively.

Sampled time series for membrane potential are calculated when $k_{2}$ are chosen as different values with $I=0, k_{1}=0.2$, and $k_{3}=0.3$, and results are shown in Figure 6. In Figure 6, multiple period bursting and chaotic bursting are obtained with $k_{2}$ being selected carefully.

In addition, extensive numerical simulations have confirmed that different modes of bursting can be obtained when system parameters are chosen appropriately. For example, when $V_{K}=-300$ is selected, and the rest parameters are the same as that in Figure 4, a type of "Fold/Hopf bursting" [45] is observed. Sampled time series for membrane potential and phase diagrams are shown in Figure 7, it is obvious that the bursting is significantly different from that in Figure 4.

3.2 Synchronization in Coupled Bursting Neurons. In this subsection, synchronization problems are studied, and two electrically coupled bursting neurons are defined by

$$
\begin{aligned}
\dot{V}_{1}= & -g_{C a} M_{\infty}\left(V_{1}\right)\left(V_{1}-V_{C a}\right)-g_{K} W_{1}\left(V_{1}-V_{K 1}\right) \\
& -g_{l}\left(V_{1}-V_{l}\right)-I_{s t}+k_{1}\left(\alpha+\beta \varphi_{1}^{2}\right) V_{1}+g_{s}\left(V_{2}-V_{1}\right), \\
\dot{W}_{1}= & \lambda\left(V_{1}\right)\left(W_{\infty}\left(V_{1}\right)-W_{1}\right), \\
\dot{\varphi}_{1}= & k_{2} V_{1}+k_{3} \varphi_{e x t}, \\
\dot{V}_{2}= & -g_{C a} M_{\infty}\left(V_{2}\right)\left(V_{2}-V_{C a}\right)-g_{K} W_{2}\left(V_{2}-V_{K 2}\right) \\
& -g_{l}\left(V_{2}-V_{l}\right)-I_{s t}+k_{1}\left(\alpha+\beta \varphi_{2}^{2}\right) V_{2}+g_{s}\left(V_{1}-V_{2}\right), \\
\dot{W}_{2}= & \lambda\left(V_{2}\right)\left(W_{\infty}\left(V_{2}\right)-W_{2}\right), \\
\dot{\varphi}_{2}= & k_{2} V_{2}+k_{3} \varphi_{e x t},
\end{aligned}
$$

where $g_{s}$ is coupling strength, and the rest parameters are the same as that in Figures 4 and 7 except for $V_{K 1}$ and $V_{K 2}$. The values of $V_{K 1}$ and $V_{K 2}$ determine the types of bursting of two neurons.

At first, synchronization transition of two identical coupled neurons is studied with $V_{K 1}=V_{K 2}=-0.7$, i.e., two coupled "Fold/Homoclinic bursting" neurons. The path to synchronization is shown in Figure 8 for different coupling strength. In Figure 8(a), two neurons present anti-phase bursting synchronization, in which neuron 1 shows period- 3 bursting and neuron 2 shows two burst patterns alternately. For $g_{s}=0.004$ in Figure 8(b), two neurons present anti-phase bursting synchronization, while neuron 1 shows chaotic bursting which is different from that in Figure 8(a). As $g_{s}$ increases, such as in Figures $8(\mathrm{c})$ and $8(\mathrm{~d})$, the two neurons show a phase-locked bursting synchronization, i.e., $1: 3$ and $1: 2$, respectively. As $g_{s}$ further increases, nearly synchronization occurs with only one spike in a burst, as shown in Figure 8(e). When the coupling strength is large enough as in Figure 8(f), the two neurons have a complete bursting synchronization. Moreover, phase diagrams of membrane potentials for two identical coupled neurons with different coupling intensity values are detected in Figure 9.

Let $V_{K 1}=-0.7$ and $V_{K 2}=-300$, the two uncoupled neurons have different bursting types, i.e., "Fold/Homoclinic bursting" and "Fold/Homoclinic bursting" respectively, and the path to synchronization for two no-identical neurons is shown in Figure 10. When the values of coupling strength are small, the two neurons show anti-phase bursting 


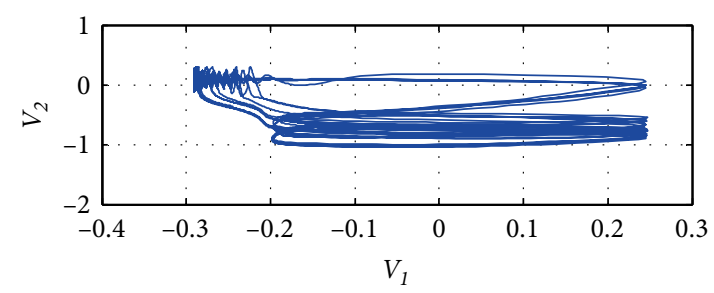

(a)

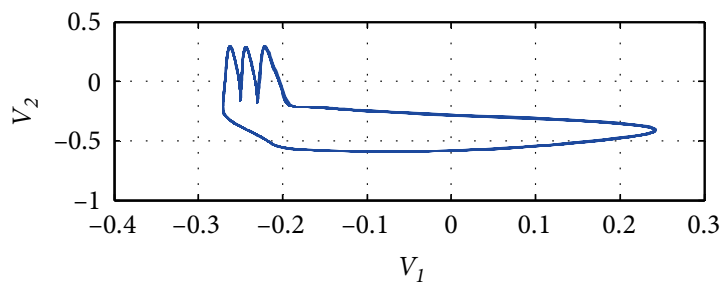

(c)

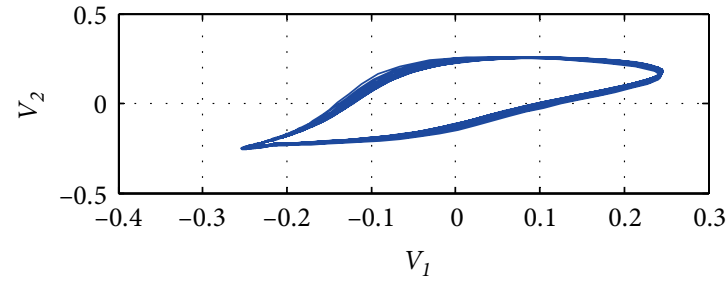

(e)

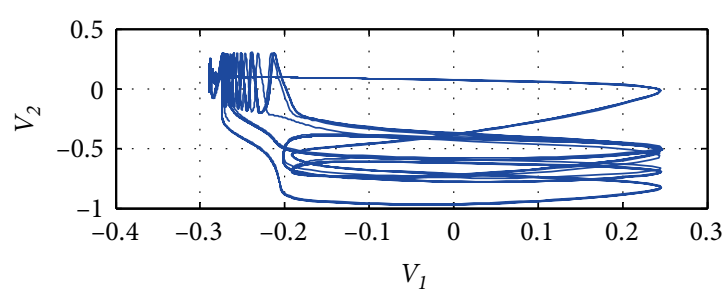

(b)

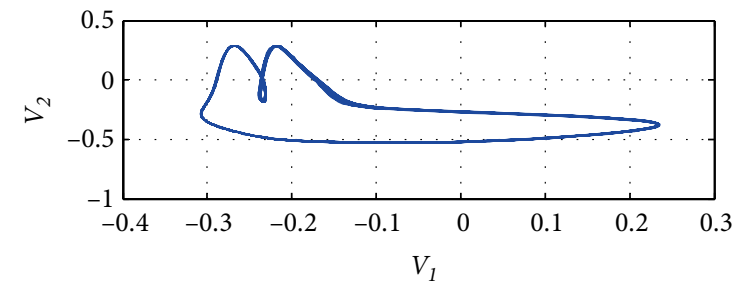

(d)

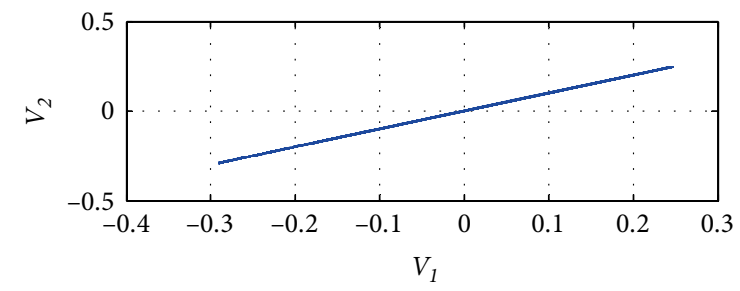

(f)

FIGURE 9: Phase diagrams of membrane potentials for two identical coupled neurons with different coupling intensity values. (a) $g_{s}=0.001$, (b) $g_{s}=0.004,(\mathrm{c}) g_{s}=0.025,(\mathrm{~d}) g_{s}=0.09,(\mathrm{e}) g_{s}=0.31$, and (f) $g_{s}=1.0$. Initial conditions are selected as $(-0.1,0,-2.5)$ and $(-0.2,0,-2.5)$, respectively.

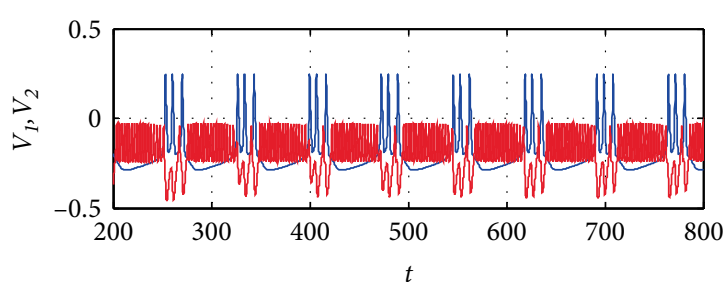

(a)

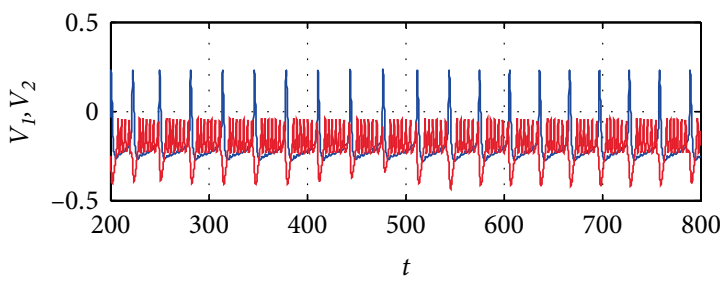

(c)

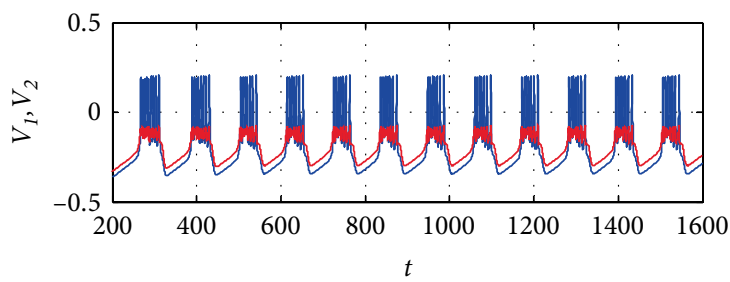

(e)

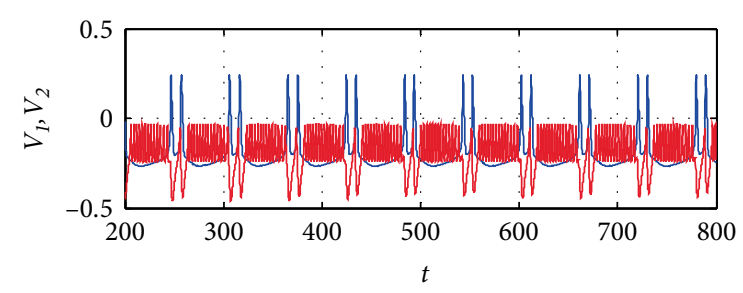

(b)

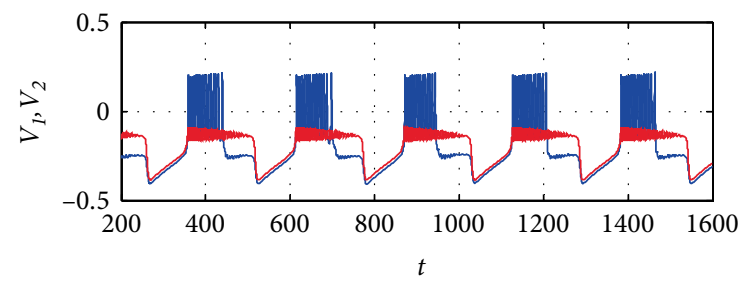

(d)

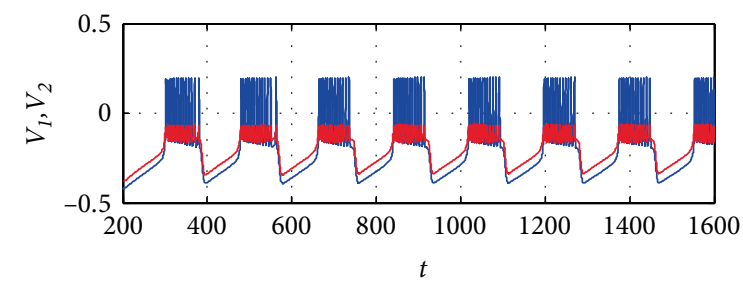

(f)

FIGURE 10: Synchronization transition of two nonidentical coupled neurons with different coupling intensity values. (a) $g_{s}=0.01$, (b) $g_{s}=0.03$, (c) $g_{s}=0.15$, (d) $g_{s}=0.5$, (e) $g_{s}=0.7$, and (f) $g_{s}=0.9$. Initial conditions are selected as $(-0.1,0,-2.5)$ and $(-0.2,0,-2.5)$, respectively. 


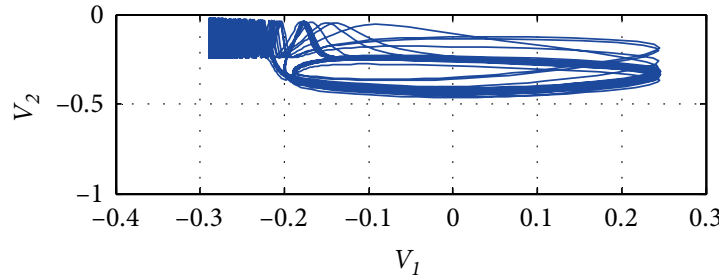

(a)

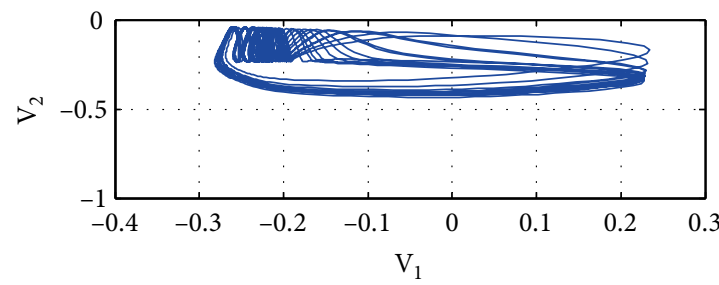

(c)

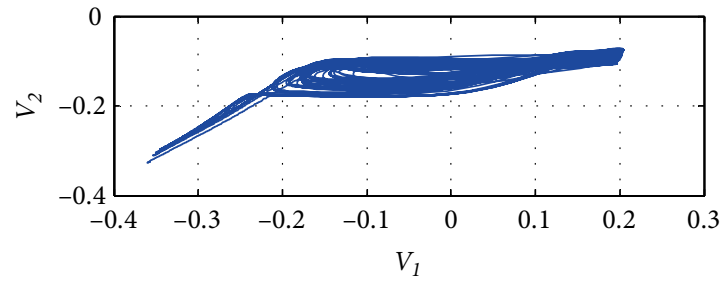

(e)

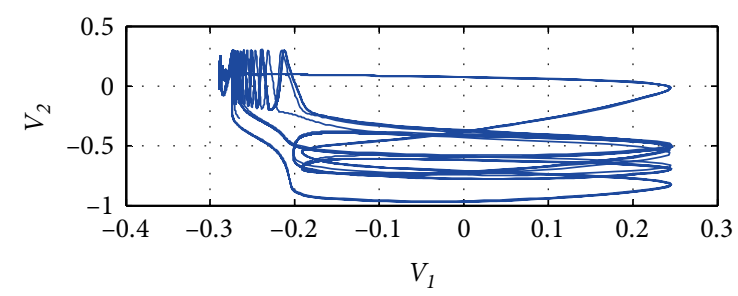

(b)

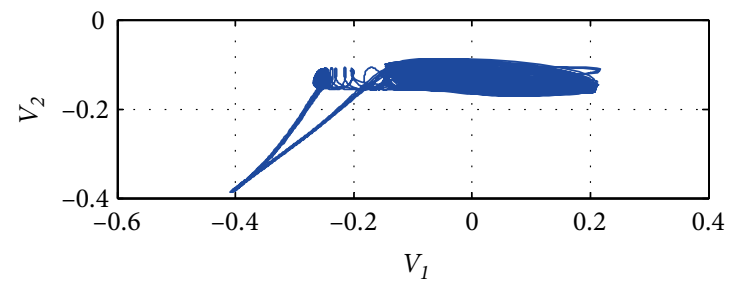

(d)

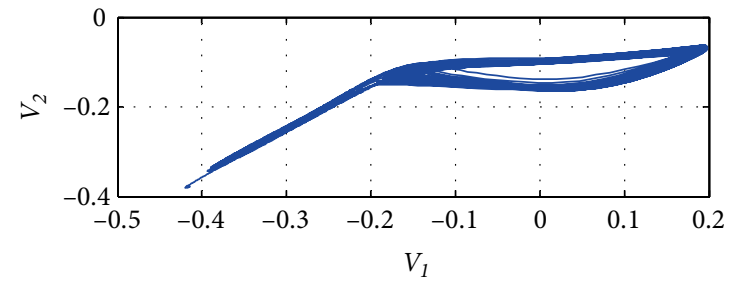

(f)

FIGURE 11: Phase diagrams of membrane potentials of two nonidentical coupled neurons with different coupling intensity values. (a) $g_{s}=0.01$, (b) $g_{s}=0.03$, (c) $g_{s}=0.15$, (d) $g_{s}=0.5$, (e) $g_{s}=0.7$, and (f) $g_{s}=0.9$. Initial conditions are selected as $(-0.1,0,-2.5)$ and $(-0.2,0,-2.5)$, respectively.

synchronization as shown in Figures 10(a)-10(c), respectively. It is worth noting that spikes in each burst for two neurons decrease as the values of coupling strength increase. When the coupling strength increases to $g_{s}=0.5$ in Figure 10(d) the two neurons have an in-phase synchrony with their own natural bursting types. As the coupling strength further increases, the waveform for neuron 2 distorts as shown in Figure 10(e), and the two neurons reach in-phase bursting synchronization, in which the two neurons have different burst amplitude. Extensive numerical simulations have confirmed that the two no-identical coupled neurons cannot attain complete synchronization whatever the values of coupling strength are selected. Furthermore, synchronization transition of phase diagrams for two nonidentical coupled neurons with different coupling intensity values are detected, and the results are shown in Figure 11.

\section{Conclusions}

In this paper, an improved third-order Morris-Lecar neuron model is proposed with the effect of electromagnetic radiation being considered, in which the bursting activities can be produced. Extensive numerical simulation results show that bursting activities of the neuron shows a deep dependence on system parameters, external forcing current and electromagnetic radiation parameters. For certain parameters, the improved Morris-Lecar neuron model can present two typical modes of bursting, i.e., "Fold/Homoclinic bursting" and "Fold/Hopf bursting", which are studied through time series and phase diagrams. Furthermore, Synchronization transitions in two electrically coupled identical as well as no-identical neurons are studied. For the identical coupled neurons, the two neurons experience a complex path to synchronization, for example, anti-phase bursting synchronization, phase-locked bursting synchronization, and complete synchronization. For no-identical coupled neurons, the two neurons only can reach anti-phase synchronization and in-phase synchronization with appropriate coupling strengths.

Bursting is an important firing category of a neuron, which occurs in different regions of neural system in the brain. It has been observed experimentally that the synchronized firing modes during sleeping of human are a kind of bursting activities of neurons [10]. Researchers [51] also argued that some neurological disorders are associate with abnormal bursting firing activity of some specified neurons. In addition, human body is imposed into the environment full of electromagnetic field, and the electromagnetic radiation is getting stronger in modern society. It has been reported that electromagnetic radiation is highly related to neuronal pathological functions, e.g., heart disease, Alzheimer's disease, and Parkinson's disease $[52,53]$. Therefore, it is very significant to study the effect of electromagnetic radiation on firing activities of neuron and collective behaviours in neuronal network, which will help us deepen the understanding of response mechanism of neural system to external environment. 


\section{Data Availability}

The data used to support the findings of this study are available from the corresponding author upon request.

\section{Conflicts of Interest}

The authors declare that they have no conflicts of interest.

\section{Acknowledgments}

This work is partially supported by the Fundamental Research Funds for the Central Universities (Grant No. 3102018KY0308) and the National Nature Science Foundation of China (Grant No. 51877162).

\section{References}

[1] A. L. Hodgkin, and A. F. Huxley, "A quantitative description of membrane current and its application to conduction and excitation in nerve," The Journal of Physiology, vol. 117, no. 4, pp. 500-544, 1952.

[2] J. L. Hindmarsh, R. M. Rose, "A model of neural bursting using three couple first order differential equations," Proceedings of the Royal Society B. Biological Sciences, vol. 221, no. 1222, pp. 87-102, 1984.

[3] C. Morris and H. Lecar, "Voltage oscillations in the barnacle giant muscle fiber," Biophysical Journal, vol. 35, no. 1, pp. 193213, 1981.

[4] J. Nagumo and S. Sato, "On a response characteristic of a mathematical neuron model," Kybernetik, vol. 10, no. 3, pp. 155-164, 1972.

[5] E. M. Izhikevich, "Which model to use for cortical spiking neurons?," IEEE Transactions on Neural Networks, vol. 15, no. 5, pp. 1063-1070, 2004.

[6] A. V. M. Herz, T. Gollisch, C. K. Machens, and D. Jaeger, "Modeling single-neuron dynamics and computations: a balance of detail and abstraction," Science, vol. 314, no. 5796, pp. 80-85, 2006.

[7] H. Gu, B. Pan, G. Chen, and L. Duan, "Biological experimental demonstration of bifurcations from bursting to spiking predicted by theoretical models," Nonlinear Dynamics, vol. 78, no. 1, pp. 391-407, 2014.

[8] J. L. Hindmarsh and R. M. Rose, "A model of the nerve impulse using two first-order differential equations," Nature, vol. 296, no. 5853, pp. 162-164, 1982.

[9] H. Gu and B. Pan, "A four-dimensional neuronal model to describe the complex nonlinear dynamics observed in the firing patterns of a sciatic nerve chronic constriction injury model," Nonlinear Dynamics, vol. 81, no. 4, pp. 2107-2126, 2015.

[10] D. A. Mccormick and H. R. Feeser, "Functional implications of burst firing and single spike activity in lateral geniculate relay neurons," Neuroscience, vol. 39, no. 1, pp. 103-113, 1990.

[11] H. Wang, J. Ma, Y. Chen, and Y. Chen, "Effect of an autapse on the firing pattern transition in a bursting neuron," Communications in Nonlinear Science and Numerical Simulation, vol. 19, no. 9, pp. 3242-3254, 2014.
[12] H. Wang, L. Wang, Y. Chen, and Y. Chen, "Effect of autaptic activity on the response of a Hodgkin-Huxley neuron," Chaos: An Interdisciplinary Journal of Nonlinear Science, vol. 24, no. 3, p. 033122, 2014.

[13] Q. Wang, M. Perc, Z. Duan, and G. Chen, "Synchronization transitions on scale-free neuronal networks due to finite information transmission delays," Physical Review E, vol. 80, no. 2, 2009.

[14] Q. Wang, Z. Duan, M. Perc, and G. Chen, "Synchronization transitions on small-world neuronal networks: Effects of information transmission delay and rewiring probability," $E P L$ (Europhysics Letters), vol. 83, no. 5, p. 50008, 2008.

[15] X. L. Song, C. N. Wang, J. Ma, and J. Tang, "Transition of electric activity of neurons induced by chemical and electric autapses," Science China Technological Sciences, vol. 58, no. 6, pp. 1007-1014, 2015.

[16] H. Gu and Z. Zhao, "Dynamics of time delay-induced multiple synchronous behaviors in inhibitory coupled neurons," PLoS One, vol. 10, no. 9, p. e0138593, 2015.

[17] B. Jia, Y. Wu, D. He, B. Guo, and L. Xue, "Dynamics of transitions from anti-phase to multiple in-phase synchronizations in inhibitory coupled bursting neurons," Nonlinear Dynamics, vol. 93, no. 3, pp. 1599-1618, 2018.

[18] Y. Gong, Y. Xie, X. Lin, and Y. Hao, "Non-Gaussian noiseoptimized intracellular cytosolic calcium oscillations," Biosystems, vol. 103, no. 1, pp. 13-17, 2011.

[19] B. Lindner, "Effects of noise in excitable systems," Physics Reports, vol. 392, no. 6, pp. 321-424, 2004.

[20] A. Bogaard, J. Parent, M. Zochowski, and V. Booth, "Interaction of cellular and network mechanisms in spatiotemporal pattern formation in neuronal networks," Journal of Neuroscience, vol. 29, no. 6, pp. 1677-1687, 2009.

[21] Q. Wang, G. Chen, and M. Perc, "Synchronous bursts on scalefree neuronal networks with attractive and repulsive coupling," PLoS One, vol. 6, no. 1, p. e15851, 2011.

[22] Z. Zhao and H. Gu, "The influence of single neuron dynamics and network topology on time delay-induced multiple synchronous behaviors in inhibitory coupled network," Chaos, Solitons \& Fractals, vol. 80, pp. 96-108, 2015.

[23] J. Best, A. Borisyuk, J. Rubin, D. Terman, and M. Wechselberger, "The dynamic range of bursting in a model respiratory pacemaker network," SIAM Journal on Applied Dynamical Systems, vol. 4, no. 4, pp. 1107-1139, 2005.

[24] H. Wang and Y. Chen, "Spatiotemporal activities of neural network exposed to external electric fields," Nonlinear Dynamics, vol. 85, no. 2, pp. 881-891, 2016.

[25] J. Li, S. Liu, W. Liu, Y. Yu, and Y. Wu, "Suppression of firing activities in neuron and neurons of network induced by electromagnetic radiation," Nonlinear Dynamics, vol. 83, no. 1-2, pp. 801-810, 2015.

[26] J. A. Robertson, J. Théberge, J. Weller, D. J. Drost, F. S. Prato, and A. W. Thomas, "Low-frequency pulsed electromagnetic field exposure can alter neuroprocessing in humans," Journal of the Royal Society Interface, vol. 7, no. 44, pp. 467-473, 2010.

[27] X. Hu, C. Liu, L. Liu, J. Ni, and Y. Yao, "Chaotic dynamics in a neural network under electromagnetic radiation," Nonlinear Dynamics, vol. 91, no. 3, 1, pp. 1541-1554, 2017.

[28] L. Chua, V. Sbitnev, and H. Kim, "Hodgkin-Huxley axon is made of memristors," International Journal of Bifurcation \& Chaos, vol. 22, no. 3, p. 1230011, 2012. 
[29] A. M. Hegab, N. M. Salem, A. G. Radwan, and L. Chua, "Neuron model with simplified memristive ionic channels," International Journal of Bifurcation and Chaos, vol. 25, no. 6, p. 1530017, 2015.

[30] M. P. Sah, H. Kim, A. Eroglu, and L. Chua, "Memristive model of the barnacle giant muscle fibers," International Journal of Bifurcation and Chaos, vol. 26, no. 1, 1630001 pages, 2016.

[31] N. Axmacher, F. Mormann, G. Fernandez, C. E. Elger, and J. Fell, "Memory formation by neuronal synchronization," Brain Research Reviews, vol. 52, no. 1, pp. 170-182, 2006.

[32] Q. Li, S. Tang, H. Zeng, and T. Zhou, "On hyperchaos in a small memristive neural network," Nonlinear Dynamics, vol. 78, no. 2, pp. 1087-1099, 2014.

[33] V. T. Pham, S. Jafari, S. Vaidyanathan, C. Volos, and X. Wang, "A novel memristive neural network with hidden attractors and its circuitry implementation," Science China Technological Sciences, vol. 59, no. 3, pp. 358-363, 2015.

[34] M. Lv and J. Ma, "Multiple modes of electrical activities in a new neuron model under electromagnetic radiation," Neurocomputing, vol. 205, pp. 375-381, 2016.

[35] M. Lv, C. Wang, G. Ren, J. Ma, and X. Song, "Model of electrical activity in a neuron under magnetic flow effect," Nonlinear Dynamics, vol. 85, no. 3, pp. 1479-1490, 2016.

[36] F. Wu, C. Wang, Y. Xu, and J. Ma, "Model of electrical activity in cardiac tissue under electromagnetic induction," Scientific Reports, vol. 6, no. 1, 2016.

[37] J. Ma, F. Wu, and C. Wang, "Synchronization behaviors of coupled neurons under electromagnetic radiation," International Journal of Modern Physics B, vol. 31, no. 2, p. 1650251, 2017.

[38] Y. Wang, J. Ma, Y. Xu, F. Wu, and P. Zhou, “The electrical activity of neurons subject to electromagnetic induction and gaussian white noise," International Journal of Bifurcation and Chaos, vol. 27, no. 2, p. 1750030, 2017.

[39] F. Wu, C. Wang, W. Jin, and J. Ma, "Dynamical responses in a new neuron model subjected to electromagnetic induction and phase noise," Physica A: Statistical Mechanics and its Applications, vol. 469, pp. 81-88, 2017.

[40] J. Wu , Y. Xu , and J. Ma , "Lévy noise improves the electrical activity in a neuron under electromagnetic radiation," PLoS One, vol. 12, no. 3, p. e0174330, 2017.

[41] G. Ren, Y. Xu, and C. Wang, "Synchronization behavior of coupled neuron circuits composed of memristors," Nonlinear Dynamics, vol. 88, no. 2, pp. 893-901, 2017.

[42] Y. Xu, Y. Jia, J. Ma, T. Hayat, and A. Alsaedi, “Collective responses in electrical activities of neurons under field coupling," Scientific Reports, vol. 8, no. 1, Article ID 1349, 2018.

[43] J. Ma, L. Mi, P. Zhou, Y. Xu, and T. Hayat, "Phase synchronization between two neurons induced by coupling of electromagnetic field," Applied Mathematics and Computation, vol. 307, pp. 321-328, 2017.

[44] M. Lv, J. Ma, Y. G. Yao, and F. Alzahrani, "Synchronization and wave propagation in neuronal network under field coupling," Science China Technological Sciences, vol. 62, no. 3, pp. 448-457, 2019.

[45] E. M. Izhikevich, "Neural excitability, spiking and bursting," International Journal of Bifurcation \& Chaos, vol. 10, no. 06, pp. 1171-1266, 2000.

[46] R. K. Upadhyay and A. Mondal, "Synchronization of bursting neurons with a slowly varying d. c. current," Chaos, Solitons \& Fractals, vol. 99, pp. 195-208, 2017.
[47] H. Wang, Q. Lu, and Q. Wang, "Bursting and synchronization transition in the coupled modified ML neurons," Communications in Nonlinear Science and Numerical Simulation, vol. 13, no. 8, pp. 1668-1675, 2008.

[48] J. Ma, Y. Wang, C. Wang, Y. Xu, and G. Ren, "Mode selection in electrical activities of myocardial cell exposed to electromagnetic radiation," Chaos, Solitons \& Fractals, vol. 99, pp. 219-225, 2017.

[49] F. Zhan and S. Liu, "Response of Electrical Activity in an Improved Neuron Model under Electromagnetic Radiation and Noise," Front ComputationalNeuroscience, vol. 11, p. 107, 2017.

[50] Y. Zhang, F. Wu, C. Wang, and J. Ma, "Stability of target waves in excitable media under electromagnetic induction and radiation," Physica A: Statistical Mechanics and its Applications, vol. 521, pp. 519-530, 2019.

[51] T. Ljungberg, P. Apicella, and W. Schultz, "Responses of monkey dopamine neurons during learning of behavioral reactions," Journal of Neurophysiology, vol. 67, no. 1, pp. 145-163, 1992.

[52] K. K. Kesari , M. H. Siddiqui , R. Meena , H. N. Vema, and S. Kumar, "Cell phone radiation exposure on brain and associated biological systems," Indian Journal of Experimental Biology, vol. 51, no. 3, pp. 187-200, 2013.

[53] C. Johansen, "Electromagnetic fields and health effectsepidemiologic studies of cancer, diseases of the central nervous system and arrhythmia-related heart disease," Scandinavian Journal of Work Environment \& Health, vol. 30, no. 1, pp. 1-30, 2004. 


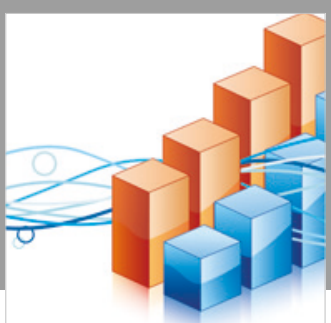

Advances in

Operations Research

\section{-n-m}
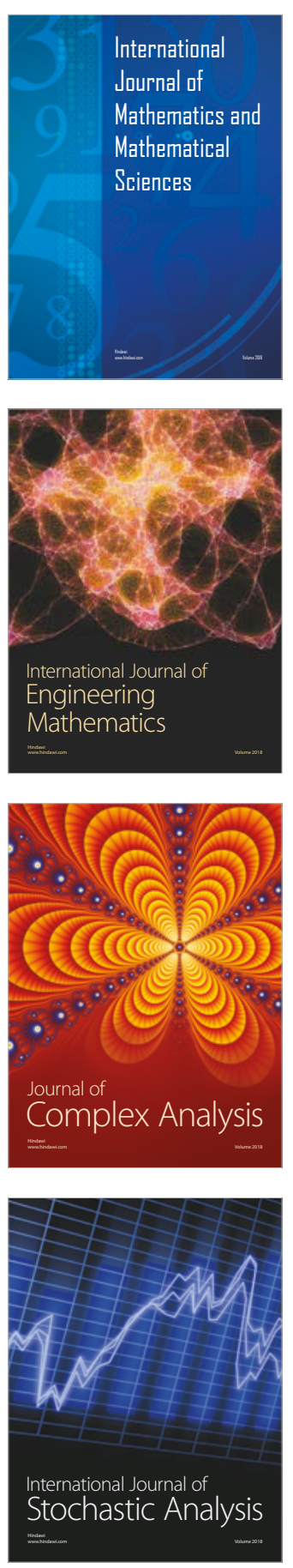
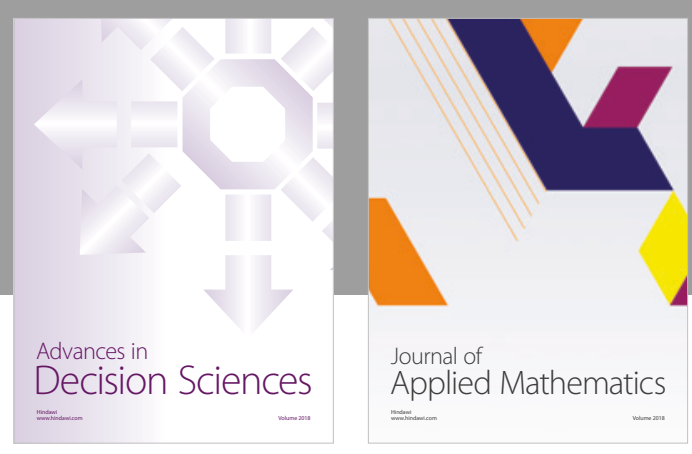

Journal of

Applied Mathematics
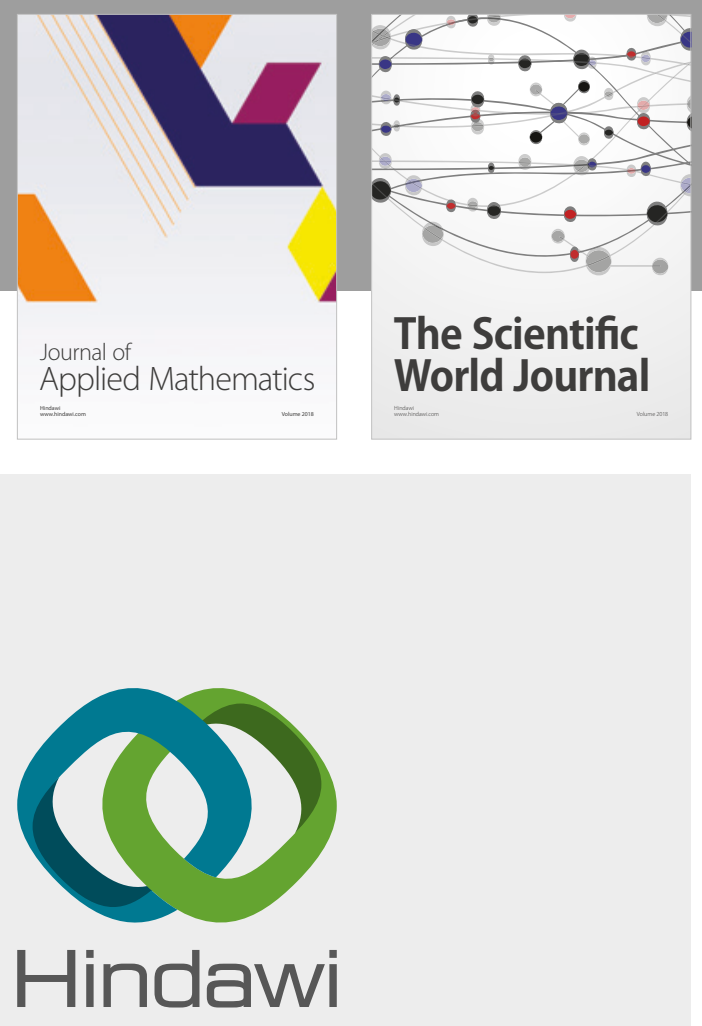

Submit your manuscripts at

www.hindawi.com

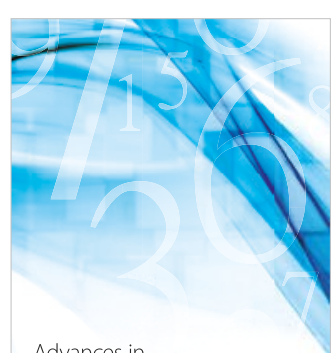

Advances in
Numerical Analysis
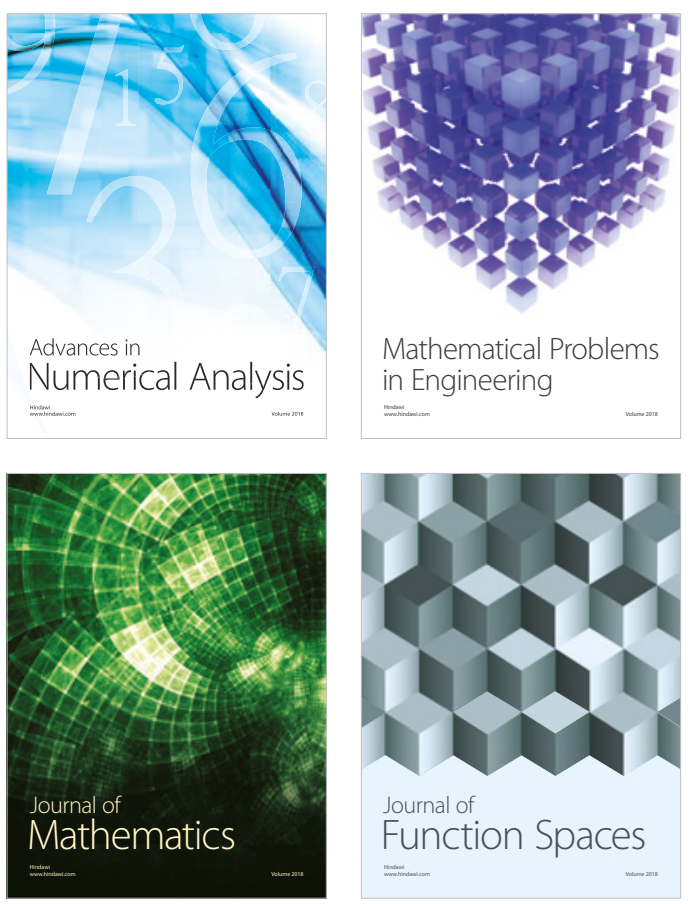

Mathematical Problems in Engineering

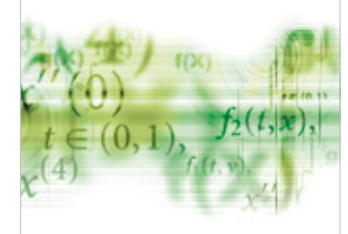

International Journal of

Differential Equations

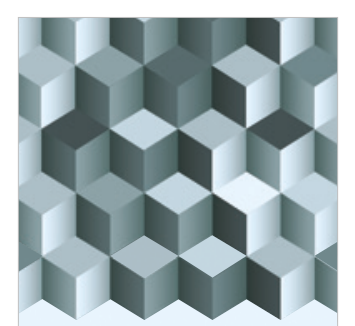

Journal of

Function Spaces
The Scientific

World Journal

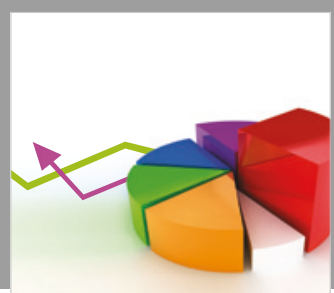

Journal of

Probability and Statistics
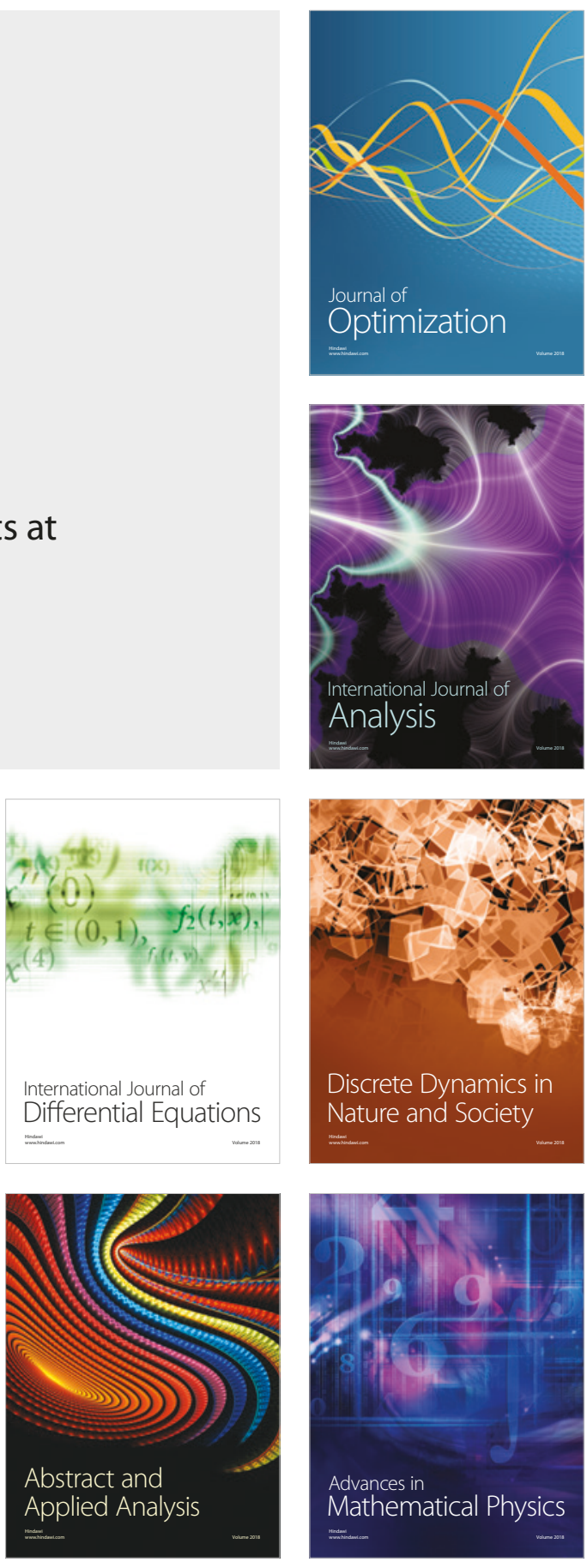